\title{
DAE $\delta$ ALUS: using multiple accelerators to determine CP violation in the neutrino sector
}

\section{Roger BARLOW*}

University of Huddersfield

for the DAESALUS Collaboration

E-mail: Roger.Barlow@cern.ch

The CP violating phase $\delta$ in the neutrino sector could be the key to the matter/antimatter asymmetry of the universe. Recent measurements indicate that $\theta_{13}$ is large, which opens the prospect of measuring $\delta$ through muon-electron neutrino mixing, with a muon neutrino beam giving an electron in the detector. The DAE $\delta$ ALUS collaboration proposes to use a 300 kton Gadolinium loaded Cherenkov detector at the DUSEL facility, and to install accelerators at three different distances to generate muon antineutrinos from stopping muons. This enables the measurement of the wavelength and amplitude of the $\bar{v}_{\mu} \rightarrow \bar{v}_{e}$ oscillation, allowing a determination of $\delta$.

XXIst International Europhysics Conference on High Energy Physics

21-27 July 2011

Grenoble, Rhône-Alpes France

${ }^{*}$ Speaker. 


\section{The experiment}

Measurement of $\delta$, the $\mathrm{CP}$ violating phase in the neutrino mixing matrix, is a crucial next step in our understanding of the universe. It appears as a $\pm \sin \delta$ term in the expression for the rate of oscillation from $v_{\mu}$ to $v_{e}$ (writing $\Delta_{i j}=\Delta m_{i j}^{2} \frac{L}{4 E_{v}}$ ):

$$
\begin{aligned}
P\left(v_{\mu}\right. & \left.\rightarrow v_{e}\right)=\sin ^{2} \theta_{23} \sin ^{2} 2 \theta_{13} \sin ^{2} \Delta_{31} \mp \sin \delta \sin 2 \theta_{13} \sin 2 \theta_{23} \sin 2 \theta_{12} \sin ^{2} \Delta_{31} \sin \Delta_{21} \\
& +\cos \delta \sin 2 \theta_{13} \sin 2 \theta_{23} \sin 2 \theta_{12} \sin \Delta_{31} \cos \Delta_{31} \sin \Delta_{21}+\cos ^{2} \theta_{23} \sin ^{2} 2 \theta_{12} \sin ^{2} \Delta_{21}
\end{aligned}
$$

where the negative and positive signs refer to the neutrino or antineutrino process respectively. This difference is a manifest sign of $\mathrm{CP}$ violation, but $\delta$ can be determined by measuring either of these rates, as the other angles and quantitites are, or soon will be, well measured.The antineutrino rate can be measured with good precision.

DAE $\delta$ ALUS (Decay At rest Experiment for $\delta_{C P}$ studies At the Laboratory for Underground Science)[1,2] proposes to do this, measuring the oscillations through the dependence on the $\Delta$ terms. This is normally done through the energy dependence, but we propose to vary $L$, maintaining strict control of $E$ by using decay-at-rest $\mu^{+}$mesons, which provide a source of muon antineutrinos up to $53 \mathrm{MeV}$, with very low electron antineutrino contamination.

For a good measurement one needs three values of $L$ corresponding to $0, \pi / 4$ and $\pi / 2$ of an oscillation, to establish the total rate, the oscillation wavelength, and the amplitude, respectively. Rather than building a source and three detectors, we propose a single detector and three sources.

\section{The Detector}

We propose a large (300 kiloton) Gadolinium loaded water Cerenkov, shared with LBNE at the underground DUSEL facility. Electron antineutrinos are detected by Inverse Beta Decay: $\overline{v_{e}} p \rightarrow e^{+} n$ on free protons. Phototubes detect these events as coincidences between the Cherenkov light from the positron and the photon from neutron absorption on Gadolinium: ${ }^{157} G d(n, \gamma)$ has kilobarn cross section. This signal has a very small background, which is measurable from 'beamoff' data. By running each accelerator with a $20 \%$ duty cycle, staggered, the event time can be used to determine the source that produced any event..

\section{The Accelerators}

Muons come from pion decay, and to produce pions requires protons of energy of order $1 \mathrm{GeV}$. The three accelerators will be located on the surface above DUSEL, $1.5 \mathrm{~km}$ from the detector, at 8 $\mathrm{km}$ and at $20 \mathrm{~km}$ distance. Several milliamps of current will be needed, so the accelerator and the Carbon target must run at several Megawatts. The requirements are similar to those of proposed Accelerator Driven Subcritical Reactor systems, and there are opportunities for synergies in their development.

The isochronous proton Cyclotron at the Paul Scherrer Institut (PSI) Switzerland, provides 590 $\mathrm{MeV}$ protons at a peak intensity of $2.4 \mathrm{~mA}$ ( $2.2 \mathrm{~mA}$ production). This is a very well-understood and successful machine[3]. It is not quite powerful enough for this purpose, but it provides a good starting point to a design. 
The Stacked Cyclotron [4] takes the PSI design and clones it several times, perhaps 5, to get the desired current. These rings are stacked one above the other, with the same magnetic field running through all of them.

The Compact Superconducting Cyclotron has been developed for proton therapy. The Monarch 250, developed by Still River Systems, is highly compact, thanks to use of a 10T Superconducting coil. However the energy is only $250 \mathrm{MeV}$, and the currents are much lower than our requirements, and it is not clear that this design can be scaled up.

The FFAG is an obvious candidate for a high power proton driver, and the principle of the nsFFAG [5] has now been proven. Application to proton acceleration at the beams and energies required is a possibility, but it has yet to be demonstarted.

Our baseline design is a Multi Megawatt Cyclotron [6] accelerating $H_{2}^{+}$ions to $1.6 \mathrm{GeV}$. Use of the charged hydrogen molecule enables extraction to be done by stripping. The binding energy is $2.75 \mathrm{eV}$, significantly larger than the $0.75 \mathrm{eV}$ of $H^{-}$, so much more stable and easier to handle. This extraction halves the energy per particle but it also doubles the beam current, so we consider $1.6 \mathrm{GeV} 2 \mathrm{~mA}$ accelerator, giving $4 \mathrm{~mA}$ of $800 \mathrm{MeV}$ protons. Two (or more) injection systems are used, to limit the space charge problems, and these are merged on entry to the main cyclotron.

\section{DAE $\delta$ ALUS and LBNE}

\begin{tabular}{|c|c|c|}
\hline & DAE $\delta$ ALUS & LBNE \\
\hline Measures & Antineutrinos & Nneutrinos (mostly) \\
Baseline & Short & Long \\
Matter effects & No & Yes \\
Energy & Low narrow band, 20-53 MeV & High wide-band $300 \mathrm{MeV}-10 \mathrm{GeV}$ \\
Background & Very low & Significant \\
\hline
\end{tabular}

It is possible for the two experiments to share the same detector. Their different features are shown in the table. Running for 10 years, DAE $\delta$ ALUS can determine $\delta$ with a precision of order $20^{\circ}$, depending on its value, and that of $\theta_{13}$. In combination with LBNE this falls to $10^{\circ}$. This could be achieved by 2030 .

\section{References}

[1] J. Alonso et al, Expression of Interest for a Novel Search for CP violation in the Neutrino sector,arXiv:1006.0260v1, (2010)

[2] J.M. Conrad, The DAESAUS Experiment, arXiv:1012.4853v1, Nucl. Phys. B Proc. Supp., (2010)

[3] Y. J. Bi et al,Towards quantitative simulations of high power proton cyclotrons Phys. Rev. ST Accel. Beams 14, 054402 (2011)

[4] P. McIntyre and A. Sattarov, Superconducting Sector Magnets for a Flux-Coupled Isochronous Cyclotron Stack, IEEE Trans on Applied Superconductivity Vol 13, p1358 (2003)

[5] R. J. Barlow et al., EMMA - the world's first non-scaling FFAG, Nucl. Instr. and Meth. A 109 (2010)

[6] L. Calabretta et al, A Multi Megawatt Cyclotron Complex to Search for CP Violation in the Neutrino Sector Proc. Cyclotron 10 and arXiv 1010.1493, (2010) 